\title{
Value Sharing of Difference Differential Polynomials of Integral Functions
}

\author{
Naveenkumar S. H.
}

\begin{abstract}
In this paper, We discussed the problems of difference differential polynomials of hyper order( $\geq 1)$ integral functions share a one value \& prove some results, which generalise \& improve the work due to Y.Liu, J.P.Wang and F.H.Liu [6].
\end{abstract}

Keywords: Y.Liu, J.P.Wang, ( $\geq 1) i$

\section{INTRODUCTION AND MAIN RESULTS}

In 2015, Y.Liu, J.P.Wang and F.H.Liu[6] obtained the result as follows.

Theorem A. Let $c \in \mathrm{C}$ and $n \geq 22$ be an integer. Let $u(z)$ and $v(z)$ be two meromorphic functions with finite order. If $E_{1}\left(1, u^{n}(z) u(z+c)\right)=E_{1}\left(1 v^{n}(z) v(z+c)\right)$, then $u(z) \equiv t_{1} v(z)$ or $u(z) v(z)=t_{2}$, for some constants $t_{1}$ and $t_{2}$ that satisfy $t_{1}^{n+1}=1$ and $t_{2}^{n+1}=1$.

Proposed Methodolgy. Nevanlinna Theory is concerned to the field of Complex Analysis. Here generally all the authors make use of the uniqueness theorem based method to prove the results by using appropriate Lemmas.

Here, we denote $\Gamma_{1}$ by $\Gamma_{1}=m_{1}+m_{2}$ and $\Gamma_{2}=m_{1}+2 m_{2}$ respectively

Question 1. What may happen if we replaced the $f^{n} f(z+c)$ by $\left[P(f) \Delta_{c} f\right]^{(k)}$ in Theorem A, for transcendental entire functions.?

2010 Mathematics. Primary 30D35.

Key W. P: Nevanlinna Theory, Entire function, Difference Differential polynomials, Uniqueness. 1

Result Analysis.

Theorem 1. Take $f$ and $g$ be trancendental integral functions of order $\sigma_{2}(f)<1$, and let $c \in \mathrm{C}$. If $E_{l}(1,[P(f$ ) $\left.\left.\Delta_{c} f\right]^{(k)}\right)=E_{l}\left(1,\left[P(g) \Delta_{c} g\right]^{(k)}\right)$ and $l, m, n$ are integers carrying circumstances:

(i) $l \geq 2, n>2 \Gamma_{2}+2 \mathrm{~km}_{2}+3$;

(ii) $l=1, n>2 \Gamma_{2}+\frac{5}{2} k m_{2}+\frac{1}{2} k m_{1}+3$;

(iii) $l=0, n>2 \Gamma_{2}+5 \mathrm{~km}_{2}+3 \mathrm{~km}_{1}+9$; Hence one of the statement follow:

(i) $f(z)=\operatorname{tg}(z)$ for a constant $t$ such that $q^{d}=1$, where $d=G C D(m+1, \ldots, m+1-i, \ldots, 1)$, for $i=0,1, \ldots, m$

(ii) $f(z)$ and $g(z)$ valids the difference equation 


$$
\begin{gathered}
R\left(w_{1}, w_{2}\right)=\left(a_{m} w_{1}^{m}+a_{m-1} w_{1}^{m-1}+\ldots+a_{0}\right)\left[w_{1}(z+c)-w_{1}(z)\right]- \\
\left(a_{m} w_{2}^{m}+a_{m-1} w_{2}^{m-1}+\ldots+a_{0}\right)\left[w_{2}(z+c)-w_{2}(z)\right]
\end{gathered}
$$

\section{LEMMAS}

In the following Lemma, defined $f$ and $g$ are the Meromorphic functions.

Lemma 2.1 [17, Lemma 1]. $P(f)=a_{n} f^{n}+a_{n-1} f^{n-1}+\ldots+a_{0}$, where $a_{n}(\neq 0), a_{n-1}, \ldots, a_{0}$ are constants. Then

$$
H(s, P(f))=n H(s, f)+G(s, f) .
$$

Lemma 2.2 [16]. $p, k$ be integers. So

$$
\begin{array}{r}
N_{p}\left(s, 0 ; f^{(k)}\right) \leq H\left(s, f^{(k)}\right)-H(s, f)+N_{p+k}(s, 0 ; f)+G(r, f), \\
N_{p}\left(s, 0 ; f^{(k)}\right) \leq k \bar{N}(s, \infty ; f)+N_{p+k}(s, 0 ; f)+S(r, f)
\end{array}
$$

\section{Lemma 2.3 [13].}

$$
N\left(s, \infty ; \frac{f}{g}\right)-N\left(s, \infty ; \frac{g}{f}\right)=N(s, \infty ; f)+N(s, 0 ; g)-N(s, \infty ; g)-N(s, 0 ; f) .
$$

Lemma 2.4 [14].

$$
H(s, f(z+c))=H(s, f)+G(s, f) .
$$

Lemma 2.5. By using above Lemma 2.4 and properties of $H(s, f)$, we have

$$
\begin{aligned}
H\left(s, \Delta_{c} f\right) & =H(s, f(z+c)-f(z)) \\
& \leq H(s, f(z+c))+H(s, f(z))+G(s, f)= \\
& 2 H(s, f)+G(s, f) .
\end{aligned}
$$

Lemma 2.6 [14].

$$
m\left(s, \frac{f(z+c)}{f(z)}\right)+m\left(s, \frac{f(z)}{f(z+c)}\right)=o\left(r^{\sigma-1+\epsilon}\right)=G(s, f) .
$$

Lemma 2.7. From Theorem 1[4] and Lemma 3[11], we conclude that if $E_{l}(1 ; C)=E_{l}(1 ; D)$, where $l \geq 2$, then also the following three cases of Theorem 1[4] holds:

(i) $T(r) \leq N_{2}(s, 0 ; C)+N_{2}(r, 0 ; D)+N_{2}(s, \infty ; C)+N_{2}(s, \infty ; D)+$

$G(r)$

(ii) $C=D$, (iii) $C D=1$, where

$T(r)=\max \{H(s, C), H(s, D)\}$ and $G(s)=o\{H(s)\}$.

Lemma 2.8. Let $C_{1}(z)=P(f) \Delta_{c} f(z)$, where $\Delta_{c} f(z) \not \equiv 0$. Then we have 


$$
(n-2) H(s, f) \leq H\left(s, C_{1}\right)+G(s, f) .
$$

Proof By the above first, fifth and sixth lemmas and first fundamental theorem, we obtain

$$
\begin{aligned}
H\left(s, f^{n+1}\right) & =H\left(s, \frac{C_{1} f}{\triangle_{c} f}\right) \\
& \leq H\left(s, C_{1}\right)+H\left(s, \frac{f}{\triangle_{c} f}\right)+G(s, f) \\
& =H\left(s, C_{1}\right)+H\left(s, \frac{\triangle_{c} f}{f}\right)+G(s, f) \\
& =H\left(s, C_{1}\right)+N\left(s, \infty ; \frac{\triangle_{c} f}{f}\right)+m\left(s, \infty ; \frac{\triangle_{c} f}{f}\right)+G(s, f) \\
& \leq H\left(s, C_{1}\right)+3 H(s, f)+S(s, f)
\end{aligned}
$$

i.e., $(n-2) H(s, f) \leq H\left(s, C_{1}\right)+G(s, f)$.

3. Proof of the Theorem 1. Let $C_{1}(z)=P(f) \Delta_{c} f$ and $D_{1}(z)=P(g) \triangle_{c} g, C=C_{1}^{(k)} D=D_{1}^{(k)}$

Using (2.1) and Lemma 2.5 we get

$$
\begin{aligned}
N_{2}(s, 0 ; C) \leq & N_{2}\left(s, 0 ;\left(C_{1}\right)^{(k)}\right)+G(s, f) \\
& \leq H\left(s,\left(C_{1}\right)^{(k)}\right)-H\left(s, C_{1}\right)+N_{k+2}\left(s, 0 ; C_{1}\right)+G(s, f) \\
& =H(s, C)-(n+2) H(s, f)+N_{k+2}\left(s, 0 ; C_{1}\right)+G(s, f) .
\end{aligned}
$$

From this we get

$$
(n+2) H(s, f) \leq H(r, C)-N_{2}(s, 0 ; C)+N_{k+2}\left(s, 0 ; C_{1}\right)+G(s, f) .
$$

Again from (2.2) we have

$$
\begin{aligned}
N_{2}(s, 0 ; C) \leq & N_{2}\left(s, 0 ;\left(C_{1}\right)^{(k)}\right)+H(s, f) \\
& \leq k \bar{N}\left(s, \infty ; C_{1}\right)+N_{k+2}\left(s, 0 ; C_{1}\right)+H(s, f) .
\end{aligned}
$$

Now arise three cases.

Case 1. Let $l \geq 2$.

Sub Case 1. If possible, that (i) of Lemma 2.9 holds. Then using (3.2) we get from (3.1)

$$
\begin{aligned}
(n+2) H(s, f) & \leq N_{2}(s, 0 ; D)+N_{2}(s, \infty ; C)+N_{2}(s, \infty ; D)+N_{k+2}\left(s, 0 ; C_{1}\right)+G(s, f)+G(s, g) \\
& \leq N_{2}\left(s, 0 ;\left[P(g) \triangle_{c} g\right]^{(k)}\right)+N_{2}\left(s, \infty ;\left[P(f) \triangle_{c} f\right]^{(k)}\right)+N_{2}\left(s, \infty ;\left[P(g) \triangle_{c} g\right]^{(k)}\right) \\
& +N_{k+2}\left(s, 0 ; P(f) \triangle_{c} f\right)+G(s, f)+G(s, g) \\
& \leq N_{k+2}\left(s, 0 ; P(g) \triangle_{c} g\right)+\left(m_{1}+(k+2) m_{2}\right) \bar{N}(s, 0 ; f)+N\left(s, 0 ; \triangle_{c} f\right) \\
& +G(s, f)+G(s, g) \\
& \leq\left(m_{1}+(k+2) m_{2} H(s, g)+2 H(s, g)+\left(m_{1}+(k+2) m_{2}\right) H(s, f)+2 H(s, f)\right. \\
& +G(s, f)+G(s, g) \\
(n+2) H(s, f) & \leq\left(m_{1}+(k+2) m_{2}+2\right) H(s, g)+\left(m_{1}+(k+2) m_{2}+2\right) H(s, f)+G(s, f)+G(s, g)
\end{aligned}
$$

Similarly,

$$
\begin{gathered}
(n+2) H(s, g) \leq\left(m_{1}+(k+2) m_{2}+2\right) H(s, f)+\left(m_{1}+(k+2) m_{2}+2\right) H(s, g) \\
+G(s, f)+G(s, g) .
\end{gathered}
$$


Combining (3.3) and (3.4) we have

$$
\left\{n-2 m_{1}-2(k+2) m_{2}-2\right\}\{H(s, f)+H(s, g)\} \leq G(s, f)+G(s, g),
$$

opposing the fact that $n>2 \Gamma_{2}+2 \mathrm{~km}_{2}+3$. So, by the above ninth lemma, we have either $C D=1$ or $C=$ D.

Sub Case 2. Suppose that $C D \equiv 1$, i.e.,

$$
\left[P(f) \Delta_{c} f\right]^{(k)}\left[P(g)\left(\Delta_{c} g\right)\right]^{k} \equiv 1 .
$$

Suppose that ' $z_{0}^{\prime}$ be a root of $f$ of order $p_{1}$. Then it is root of $\left[P(f) \Delta_{f} f\right]^{(k)}$ of order $\left(5 k+p_{0}\right) p_{1}-k$, where $\left(p_{0}\right.$ $>19)$, then $z_{0}$ is pole of order $q_{1}$, then it is a point at which infinity of $\left[p(g)\left(\Delta_{c} g\right)\right]^{k}$ of order $\left(5 k+p_{0}\right) q_{1}+$ $k$.

$$
\begin{gathered}
\Rightarrow\left(5 k+p_{0}\right) p_{1}-k=\left(5 k+p_{0}\right) q_{1}+k \\
\Rightarrow\left(5 k+p_{0}\right)\left(p_{1}-q_{1}\right)=2 k
\end{gathered}
$$

which is a contradiction. Since $p_{1}, q_{1}$ and $k$ are integers and $p_{0} \geq 19$.

$\Rightarrow\left(5 k+p_{0}\right) p_{2}+k=\left(5 k+p_{0}\right) q_{2}-k$

$\Rightarrow\left(5 k+p_{0}\right)\left(q_{2}-p_{2}\right)=2 k$

which is a opposite proposition. Since $p_{2}, q_{2}$ and $k$ are integers and $p_{0} \geq 19$.

Hence $f \neq \infty$. Similarly, we can prove $g \neq \infty$. Now we can conclude that $f$ and $g$ are integral functions having no zeros \&poles.

Therefore $f(z)=e^{\alpha(z)}$ and $g(z)=e^{\beta(z)}$, where $\alpha(z)$ and $\beta(z)$ are expressions. Substituting $f$ and $g$ in (3.5),

$$
\begin{aligned}
& {\left[\left(a_{n} e^{n \alpha(z)}+a_{n-1} e^{(n-1) \alpha(z)}+\ldots+a_{0}\right)\left(e^{\alpha(z+c)}-e^{\alpha(z)}\right)\right]^{(k)}} \\
& {\left[\left(a_{n} e^{n \beta(z)}+a_{n-1} e^{(n-1) \beta(z)}+\ldots+a_{0}\right)\left(e^{\beta(z+c)}-e^{\beta(z)}\right)\right]^{(k)} \equiv 1 .}
\end{aligned}
$$

Let

$$
\begin{array}{rrr}
\alpha(z+c)=A_{n} & \alpha(z) & =B_{n} \\
\beta(z+c)=c_{n} & \beta(z) & =d_{n} .
\end{array}
$$

Similarly as above, we get contradiction.

For $k \geq 3$, as above, we deduce a opposite proposition . Therefore, the condition

$$
\left[P(f) \Delta_{c} f\right]^{(k)}\left[P(g)\left(\Delta_{c} g\right)\right]^{k} \equiv 1
$$

i.e., $F G \equiv 1$ is not possible.

Sub Case 3. Let $C \equiv D$

i.e.,

$$
\left[P(f) \Delta_{c} f(z)\right]^{(k)} \equiv\left[P(g) \Delta_{c} g(z)\right]^{(k)} .
$$


From (3.8) we get

$$
P(f) \Delta_{c} f(z) \equiv P(g) \Delta_{c} g(z)+P_{1}(z),
$$

where $P_{1}(z)$ is a expression of power maximum $k-1$. Suppose $P_{1}(z) \neq 0$. Then from (3.6)

$$
\frac{P(f) \triangle_{c} f}{P_{1}} \equiv \frac{P(g) \triangle_{c} g}{P_{1}}+1 .
$$

By Lemma 2.12 and the $2^{\text {nd }}$ fundamental theorem

$$
\begin{aligned}
(n-2) H(s, f) & \leq H\left(s, P(f) \triangle_{c} f\right)+G(s, f) \\
& \leq H\left(s, \frac{P(f) \triangle_{c} f}{P_{1}(z)}\right)+G(s, f) \\
& \leq \bar{N}\left(s, \frac{P(f) \triangle_{c} f}{P_{1}(z)}\right)+\bar{N}\left(s, \frac{P_{1}(z)}{P(f) \triangle_{c} f}\right)+\bar{N}\left(r, \frac{P_{1}(z)}{P(g) \triangle_{c} g}\right)+G(s, f)+G(s, g) \\
& \leq \bar{N}(s, P(f))+\bar{N}\left(s, \triangle_{c} f\right)+\bar{N}(s, 0 ; P(f))+\bar{N}\left(s, 0 ; \triangle_{c} f\right) \\
& +\bar{N}(s, 0 ; P(g))+\bar{N}\left(s, 0 ; \triangle_{c} g\right)+G(s, f)+G(s, g) \\
& \leq 2 H(s, f)+\Gamma_{1} H(s, f)+2 H(s, f)+\Gamma_{1} H(s, g)+2 H(s, g)+G(s, f)+G(s, g) \\
(n-2) H(s, f) & \leq\left(\Gamma_{1}+4\right) H(s, f)+\left(\Gamma_{1}+2\right) H(s, g)+G(s, f)+G(s, g) .
\end{aligned}
$$

Similarly, we have

$$
(n-2) H(s, g) \leq\left(\Gamma_{1}+4\right) H(s, g)+\left(\Gamma_{1}+2\right) H(s, f)+G(s, f)+G(s, g) .
$$

Therefore we get

$$
\begin{gathered}
(n-2)[H(s, f)+H(s, g)] \leq\left(2 \Gamma_{1}+6\right)[H(s, f)+H(s, g)]+G(s, f)+G(s, g) n[H(s, \\
f)+H(s, g)] \leq\left(2 \Gamma_{1}+8\right)[H(s, f)+H(s, g)]+G(s, f)+G(s, g)
\end{gathered}
$$

which is a contradiction since $n>2 \Gamma_{1}+9$. Hence $P_{1}(z) \equiv 0$ and so from (3.9) we obtain

$$
\begin{gathered}
P(f) \Delta_{c}(f)=P(g) \Delta_{c}(g) \\
\left(a_{m} f^{m}+a_{m+1} f^{m+1}+\ldots+a_{0}\right)(f(z+c)-f(z)) \\
=\left(a_{m} g^{m}+a_{m+1} g^{m+1}+\ldots+a_{0}\right)(g(z+c)-g(z)) .
\end{gathered}
$$

Let $h=\frac{f}{g}$.

If $h$ not varies then put $f=g h$ and $f(z+c)=g(z+c) h(z+c)$ in (3.10) we get,

$$
\begin{aligned}
& a_{m} g^{m}\left[\left(h^{m} h(z+c)-1\right) g(z+c)-\left(h^{m} h(z)-1\right) g(z)\right]+a_{m-1} g^{m-1}\left[\left(h^{m-1} h(z+c)\right.\right. \\
& \left.-1) g(z+c)-\left(h^{m-1} h(z)-1\right) g(z)\right] \\
& \quad+\ldots+a_{0}[(h(z+c)-1) g(z+c)-(h(z)-1) g(z)]=0
\end{aligned}
$$

implies $h^{d}=1$, where

$$
d=G C D(m+1, \ldots, m+1-i, \ldots, 1), \quad a_{m-i} \neq 0
$$

for $i=0,1, \ldots, m$.

Thus $f(z)=\operatorname{tg}(z)$.

If $\mathrm{h}$ varies, then $f(z)$ and $g(z)$ satisfy the difference equation 


$$
R(f, g) \equiv 0 .
$$

Case 2. Let $l=1$.

Sub Case 1. Using Lemma 2.15[2] and (3.2) we obtain from (3.1)

$$
\begin{aligned}
(n+2) H(s, f) & \leq N_{2}(s, 0 ; D)+N_{2}(s, \infty ; C)+N_{2}(s, \infty ; D)+\frac{1}{2} \bar{N}(s, 0 ; C)+\frac{1}{2} \bar{N}(s, \infty ; C)+N_{k+2}\left(s, 0 ; C_{1}\right) \\
& +G(s, f)+G(s, g) \\
& \leq\left(m_{1}+(k+2) m_{2}\right) H(s, g)+2 H(s, g)+\left(m_{1}+(k+2) m_{2}\right) H(s, f)+2 H(s, f) \\
& +\frac{1}{2} k\left(m_{1}+m_{2}\right) H(s, f)+H(s, f)+G(s, f)+G(s, g) \\
(n+2) H(s, f) & \leq\left(m_{1}+2 m_{2}+k m_{2}+2\right) H(s, g)+\left(m_{1}+2 m_{2}+\frac{3}{2} k m_{2}+\frac{1}{2} k m_{1}+2\right) H(s, f) \\
& +G(s, f)+G(s, g) .
\end{aligned}
$$

Similarly,

$$
\begin{aligned}
(n+2) H(s, g) & \leq\left(m_{1}+2 m_{2}+k m_{2}+2\right) H(s, f)+\left(m_{1}+2 m_{2}+\frac{3}{2} k m_{2}+\frac{1}{2} k m_{1}+2\right) H(s, g) \\
& +G(s, f)+G(s, g) .
\end{aligned}
$$

Combining (3.11) and (3.12) we have

$$
\left\{n-2 m_{1}-4 m_{2}-\frac{5}{2} k m_{2}-\frac{1}{2} k m_{1}-2\right\}\{H(s, f)+H(s, g)\} \leq G(s, f)+G(s, g),
$$

contradicting the fact that $n>2 \Gamma_{2}+\frac{5}{2} k m_{2}+\frac{1}{2} k m_{1}+3$. Therefore, by Lemma 2.15[2], we have either $C D$ $=1$ or $C=D$.

Sub Case 2. Then by Subcase 2 of Case $1, C D=1$ is not possible .

Sub Case 3. Let $F \equiv G$. Then the conclusion get from Subcase 3 of Case 1 .

Case 3. Let $l=0$.

Sub Case 1. By Lemma 2.14[2] and (3.2) we get from (3.1)

$$
\begin{aligned}
(n+2) H(s, f) \leq & N_{2}(s, 0 ; D)+N_{2}(s, \infty ; C)+N_{2}(s, \infty ; D)+2 N(s, 0 ; C)+N(s, 0 ; D)+N_{k+2}\left(s, 0 ; C_{1}\right) \\
& +2 \bar{N}(s, \infty ; C)+\bar{N}(s, \infty ; D)+G(s, f)+G(s, g) . \\
& \leq\left(m_{1}+(k+2) m_{2}\right) H(s, g)+2 H(s, g)+2\left(k\left(m_{1}+m_{2}\right)+2\right) H(s, f) \\
& +\left(k\left(m_{1}+m_{2}\right)+2\right) H(s, g)+\left(m_{1}+(k+2) m_{2}\right) H(s, f) \\
& +2 H(s, f)+G(s, f)+G(s, g) \\
(n+2) H(s, f) & \leq\left(m_{1}+2 m_{2}+2 k m_{2}+k m_{1}+4\right) H(s, g)+\left(m_{1}+2 m_{2}+3 k m_{2}+2 k m_{1}+6\right) H(s, f) \\
& +G(s, f)+G(s, g) .
\end{aligned}
$$

Similarly,

$$
\begin{array}{r}
(n+2) H(s, g) \leq\left(m_{1}+2 m_{2}+2 k m_{2}+k m_{1}+4\right) H(s, f)+\left(m_{1}+2 m_{2}+3 k m_{2}+2 k m_{1}+6\right) H(s, g) \\
+G(s, f)+G(s, g) .
\end{array}
$$

Combining (3.13) and (3.14) we have

$$
\left\{n-2 m_{1}-4 m_{2}-5 k m_{2}-3 k m_{1}-8\right\}\{H(s, f)+H(s, g)\} \leq G(s, f)+G(s, g),
$$

opposing the fact that $n>2 \Gamma_{2}+5 k m_{2}+3 k m_{1}+9$. Therefore, by Lemma 2.14[2], we have either $C D=1$ or $C=D$.

Then by Subcase 2 and 3 of Case 1, the result follows.

III. CONCLUSION. 
We can replace difference polynomials $f^{n} f(z+c)$ by $\left[P(f) \Delta_{c} f\right]^{(k)}$ in Theorem A, for transcendental entire functions. So that the result obtained in this paper improves and generalises the many previous results on difference polynomials.

\section{REFERENCES}

1. Banerjee, Abhijit; Chakraborty, Bikash. Further investigations on a question of Zhang and Lu“. Ann. Univ. Paedagog. Crac. Stud. Math. 14 (2015), 105-119.

2. Banerjee, Abhijit. Meromorphic functions sharing one value. Int. J. Math. Sci. 2005, no. 22, 3587-3598.

3. Hayman, W. K. Meromorphic functions. Oxford Mathematical Monographs Clarendon Press, Oxford 1964 xiv+191 pp

4. Lahiri, Indrajit. Weighted value sharing and uniqueness of meromorphic functions. Complex Variables Theory Appl. 46 (2001), no. 3, 241-253.

5. K. Liu; X. L. Liu; T. B. Cao, Some results on zeros distributions and uniqueness of derivatives of difference polynomials, (2011), http://arxiv.org/abs/1107.0773vl.

6. Liu, Y.; Wang, J. P.; Liu, F. H. Some results on value distribution of the difference operator. Bull. Iranian Math. Soc. 41 (2015), no. 3 , 603-611.

7. Laine, Ilpo. Nevanlinna theory and complex differential equations De Gruyter Studies in Mathematics, 15. Walter de Gruyter \& Co., Berlin, 1993. viii+341 pp. ISBN: 3-11-013422-5.

8. Qi, Xiao-Guang; Yang, Lian-Zhong; Liu, Kai. Uniqueness and periodicity of meromorphic functions concerning the difference operator. Comput. Math. Appl. 60 (2010), no. 6, 1739-1746.

9. $\mathrm{Xu}$, Hong Yan; Liu, Kai; Cao, Ting Bin. Uniqueness and value distribution for $q$-shifts of meromorphic functions. Math. Commun. 20 (2015), no. 1, 97-112.

10. Yi, H.X; Yang, C.C. Uniqueness Theory of Meromorphic functions,Beijing, Science Press, 1995.

11. Yang, Chung-Chun; Hua, Xinhou. Uniqueness and value-sharing of meromorphic functions. Ann. Acad. Sci. Fenn. Math. 22 (1997), no. 2, 395-406.

12. Yang, Chung-Chun; Yi, Hong-Xun. Uniqueness theory of meromorphic functions. Mathematics and its Applications, 557. Kluwer Academic Publishers Group, Dordrecht, 2003. viii+569 pp. ISBN: 1-4020-1448-1

13. Yang, L. Value distribution theory. Translated and revised from the 1982 Chinese original. Springer-Verlag, Berlin; Science Press Beijing, Beijing, 1993.

14. Yik-Man, Chiang; Feng, Shao-Ji. On the Nevanlinna characteristic of $f(z+\eta)$ and difference equations in the complex plane. Ramanujan J. 16 (2008), no. 1, 105-129.

15. Yamanoi, Katsutoshi. The second main theorem for small functions and related problems. Acta Math. 192 (2004), no. 2 , 225-294.

16. Zhang, Ji-Long; Yang, Lian-Zhong. Some results related to a conjecture of R. Bruck. JIPAM. J. Inequal. Pure Appl. Math. 8 (2007), no. 1, Article 18, 11 pp.

17. Zhang, Xiao-Yu; Chen, Jun-Fan; Lin, Wei-Chuan. Entire or meromorphic functions sharing one value. Comput. Math. Appl. 56 (2008), no. 7, 1876-1883. 